\title{
Synchronization of Heterogeneous Oscillators by Noninvasive Time-Delayed Cross Coupling
}

\author{
Thomas Jüngling and Ingo Fischer \\ Instituto de Física Interdisciplinar y Sistemas Complejos, IFISC (CSIC-UIB), Campus Universitat Illes Balears, \\ E-07122 Palma de Mallorca, Spain \\ Eckehard Schöll \\ Institut für Theoretische Physik, Technische Universität Berlin, Hardenbergstraße 36, D-10623 Berlin, Germany \\ Wolfram Just ${ }^{*}$ \\ School of Mathematical Sciences, Queen Mary University of London, Mile End Road, London E1 4NS, United Kingdom
}

(Received 18 August 2015; published 3 November 2015)

\begin{abstract}
We demonstrate that nonidentical systems, in particular, nonlinear oscillators with different time scales, can be synchronized if a mutual coupling via time-delayed control signals is implemented. Each oscillator settles on an unstable state, say a fixed point or an unstable periodic orbit, with a coupling force which vanishes in the long time limit. We present the underlying theoretical considerations and numerical simulations, and, moreover, demonstrate the concept experimentally in nonlinear electronic oscillators.
\end{abstract}

DOI: 10.1103/PhysRevLett.115.194101

PACS numbers: 05.45.Xt, 02.30.Ks

Synchronization is probably the most prominent dynamical feature in complex coupled oscillator systems, with a multitude of facets and applications in various disciplines such as engineering or biology [1]. Synchronization is often understood as identical synchronization, in particular, within the technological and the engineering context. However, the dynamical manifestation of synchronization in real world applications can show up in various disguises, e.g., appearing only in a special feature like the phase of the signal [2] or involving time lags [3]. At a very general level any motion which, due to coupling, is attracted to a submanifold in phase space can be termed as being synchronized [4].

A particularly important application of synchronization is in the area of control problems. Within the physics context such a view is vividly illustrated by what has been originally called time delay autosynchronization, the use of timedelayed feedback to stabilize periodic orbits [5]. Inspired by such schemes we tackle here the question of whether two entirely different dynamical systems have the ability to mutually stabilize each other by bidirectional coupling via time-delayed signals. Moreover, we address the aspect of whether or not this can even be achieved noninvasively which might sound counterintuitive, considering the heterogeneity of the oscillators and the dynamical states. In formal terms let us consider two different systems governed by differential equations $\dot{\boldsymbol{x}}=\boldsymbol{f}(\boldsymbol{x})$ and $\dot{\boldsymbol{y}}=\boldsymbol{g}(\boldsymbol{y})$, each of them having an unstable target state, say a periodic orbit with periods $\tau$ and $\theta$, respectively. We are going to investigate whether a mutual time-delayed coupling of the type

$$
\begin{aligned}
\dot{\boldsymbol{x}}(t) & =\boldsymbol{f}(\boldsymbol{x}(t))-K_{x}(\boldsymbol{y}(t)-\boldsymbol{y}(t-\theta)), \\
\dot{\boldsymbol{y}}(t) & =\boldsymbol{g}(\boldsymbol{y}(t))-K_{y}(\boldsymbol{x}(t)-\boldsymbol{x}(t-\tau))
\end{aligned}
$$

is able to stabilize both target states of the individual units in a noninvasive way, i.e., with the time-delayed interactions to vanish when the periodic target states are reached. While in applications the coupling of the time-delayed signal will be mediated by matrices, in particular, in cases when $\boldsymbol{x}$ and $\boldsymbol{y}$ have different dimensions, we will consider for simplicity of the argument scalar valued quantities $K_{x / y}$ for most parts of our theoretical considerations. From a pure mathematical perspective there are no obvious formal obstacles which prevent the scheme, Eq. (1), to stabilize periodic orbits of each unit. However, on physical grounds the scheme looks intriguing, as a mutual coupling would not affect the trace of the Jacobian which determines stability properties of unstable solutions. In addition, the two periods $\tau$ and $\theta$ of the target states may differ. Rather counterintuitively, we will show that the diversity of the constituents and not a resonance condition is a key criterion. In most studies of complex coupled structures disorder and diversity are often an important challenge to cope with, see, e.g., [6]. Rather than being a nuisance we have here a concept in which diversity plays a vital role for the stabilization to work.

Equation (1) is in fact a particular case of generalized synchronization, with the synchronization manifold being a torus defined by the two unstable periodic orbits of the individual units. We will tackle the synchronization problem from different sides. The stability problem emerging from Eq. (1) can be studied in special cases in closed analytic form. The analytic considerations will reveal some important mechanisms which we are then going to reinforce by numerical simulations. Our simulations of the Toda system serve as a proof of concept study and indicate the feasibility of the concept. Finally, we demonstrate the successful implementation of our ideas with 
electronic circuit experiments highlighting the robustness of the scheme in real world setups. Moreover, the experiments indicate the existence of further parameter regimes, in which local control, approximately noninvasive, of only one of the oscillators is achieved.

Linear stability in Eq. (1) is governed by the corresponding variational equation

$$
\begin{aligned}
\delta \dot{\boldsymbol{x}}(t) & =D \boldsymbol{f}(t) \delta \boldsymbol{x}(t)-K_{x}(\delta \boldsymbol{y}(t)-\delta \boldsymbol{y}(t-\theta)), \\
\delta \dot{\boldsymbol{y}}(t) & =D \boldsymbol{g}(t) \delta \boldsymbol{y}(t)-K_{y}(\delta \boldsymbol{x}(t)-\delta \boldsymbol{x}(t-\tau)),
\end{aligned}
$$

where the Jacobians $D f$ and $D \boldsymbol{g}$ inherit the $\tau$ - and $\theta$ periodic time dependence from the target orbits. In general, Eq. (2) results in a fairly advanced quasiperiodic eigenvalue problem, for which the corresponding Lyapunov exponents are not easy to study by analytic means. Only very few rigorous regularity results are available for setups which do not even include time delay, see, e.g., [7]. Nevertheless, by a simple scaling argument it is fairly obvious that the control intensities $K_{x}$ and $K_{y}$ contribute to stability not separately but only as a product $K_{x} K_{y}$, if we consider, for instance, a coupling via scalar quantities. Such a property is a consequence of the mutual coupling scheme and it has been proposed as a suitable mechanism to implement a secure communication channel [8-10].

To make some progress with simple analytic arguments and to provide a proof of concept let us consider the case where the time dependence in the Jacobian can be ignored. That is certainly the case when we consider the stabilization of fixed points. But even for periodic orbits with $\boldsymbol{f}=\boldsymbol{g}$ and a scalar coupling of the control force the time dependence can be removed by an appropriate coordinate transformation. The analysis of Eq. (2) reduces to the discussion of a transcendental eigenvalue equation. We can even decouple the eigenvalue equation if we assume all matrices to commute. The corresponding quasipolynomial finally reads

$$
(\Lambda-\mu)(\Lambda-\nu)=K_{x} K_{y}(1-\exp (-\Lambda \tau))(1-\exp (-\Lambda \theta))
$$

where $\mu$ and $\nu$ are the eigenvalues or the Floquet exponents of the Jacobians. It is fairly straightforward to check, for instance, by employing the properties of the Lambert $W$ function, that Eq. (3) possesses an unstable eigenmode if identical systems are considered, i.e., $\mu=\nu, K_{x}=K_{y}$, and $\tau=\theta$. Thus, a certain amount of diversity is required to obtain synchronization. The same caveat is likely to apply for the original time dependent stability problem, Eq. (2).

For an analytical solution of the quasipolynomial, Eq. (3), and to prove the feasibility of our concept we constrain to the special case of identical periodic orbits of period $\tau$ with negative Floquet multipliers, $\exp (\mu \tau)=\exp (\nu \tau)<-1$, a case which occurs frequently in the neighborhood of period doubling scenarios. As stated previously, the obvious choice $\tau=\theta$ results in Floquet exponents with positive real part. Hence, to supply the required diversity we choose one of the time delays as twice the proper period, $\theta=2 \tau$. Then a simple expansion of Eq. (3) for small values of $\lambda=\operatorname{Re}(\mu)$ and $K_{x} K_{y}$ yields for the leading eigenvalues

$$
z^{2}-2\left(\lambda+2 K_{x} K_{y} \tau\right) z+\lambda^{2}+\mathcal{O}\left(K_{x} K_{y} z^{2}\right)=0
$$

where we have introduced the abbreviation $\Lambda=z+i \pi / \tau$. Hence, real parts change sign and synchronization sets in if $-K_{x} K_{y} \geq \lambda /(2 \tau)$. In particular, control gains of an opposite sign are required in this case. Apart from such a perturbative treatment we can work out as well the control domain, by obtaining a parametric representation of the boundary from Eq. (3) with the choice $\Lambda=i \Omega$, see Fig. 1. It does not come as a surprise that the control interval depends sensitively on the real part of the Floquet exponent of the unstable orbit, see, e.g., [11], and that synchronization in this simple setup fails if that exponent exceeds a critical value.

Analytic approaches, as seen above, are often only applicable if one puts severe constraints on the underlying system. While numerical simulations are never able to fully prove assertions, we can demonstrate that synchronization by mutual time-delayed coupling works under conditions which have not been covered so far. For the purpose of such a demonstration we resort to driven Toda oscillators, which can be viewed as a simple, experimentally relevant, paradigmatic oscillator model [12]. Externally driven systems, unlike autonomous models, are often more robust with regard to time-delayed feedback. The equations of motion are given by

$$
\begin{aligned}
\dot{x}_{1}(t)= & x_{2}(t), \\
\dot{x}_{2}(t)= & -\gamma_{x} x_{2}(t)-\alpha_{x}\left[\exp \left(x_{1}(t)\right)-1\right]+A_{x} \sin (2 \pi t / \tau) \\
& -K_{x}\left(y_{2}(t)-y_{2}(t-\theta)\right),
\end{aligned}
$$

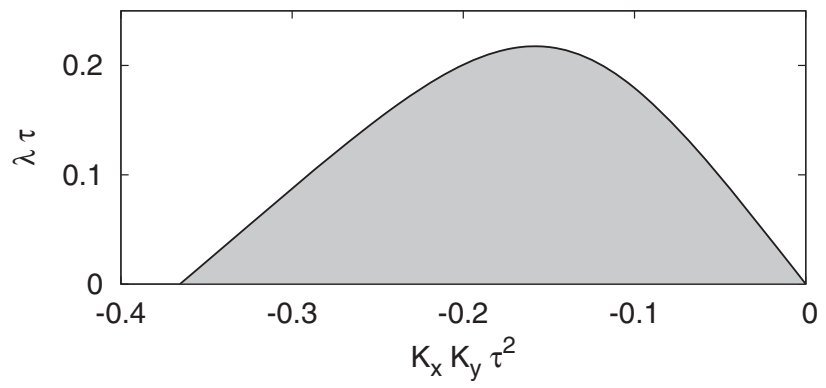

FIG. 1. Exact analytic result for the control domain of two $\tau$-periodic unstable orbits with Floquet exponents $\mu=\nu=$ $\lambda+i \pi / \tau$, diagonal coupling, and time delays $\theta=2 \tau$, according to Eq. (3). 
with the second set of equations being obtained by exchanging the labels $x$ and $y$ and the periods $\tau$ and $\theta$, see Eqs. (1). The parameter values $\gamma_{x}=\gamma_{y}=0.5, \alpha_{x}=20$, $A_{x}=33, \alpha_{y}=30$, and $A_{y}=55$ are chosen such that each oscillator without control possesses an unstable periodic orbit of period $\tau=1$ and $\theta=\tau / 1.9$ with a Floquet exponent of moderate size, see the previous section. The two oscillators admit chaotic dynamics and a stable periodtwo orbit, respectively, see Figs. 2(b) and 2(c). With control gains $K_{x}=K_{y}$ of suitable size each of the unstable orbits becomes stable and the system synchronizes with no particular phase relation between both oscillators, see Fig. 2(a). Initially, the absence of any resonance condition or phase relation is astounding, but it is mainly due to the design of the control scheme. The control forces vanish on the entire torus and thus cannot trigger any phase locking mechanism. Within the control interval the residual signal, i.e., the norm of the time-delayed differences, displays the usual exponential decay in time, see Fig. 2(d). Thus, ultimately the control becomes noninvasive. The corresponding leading nontrivial Lyapunov exponent, i.e., discarding the two trivial vanishing exponents, shows a characteristic $V$-shaped dependence on the control gain, see Fig. 2(e).

The numerical findings are robust with regard to parameter variations. The results are consistent with the analytic finding that diversity of the constituents is a major condition for the synchronization to work. Furthermore,
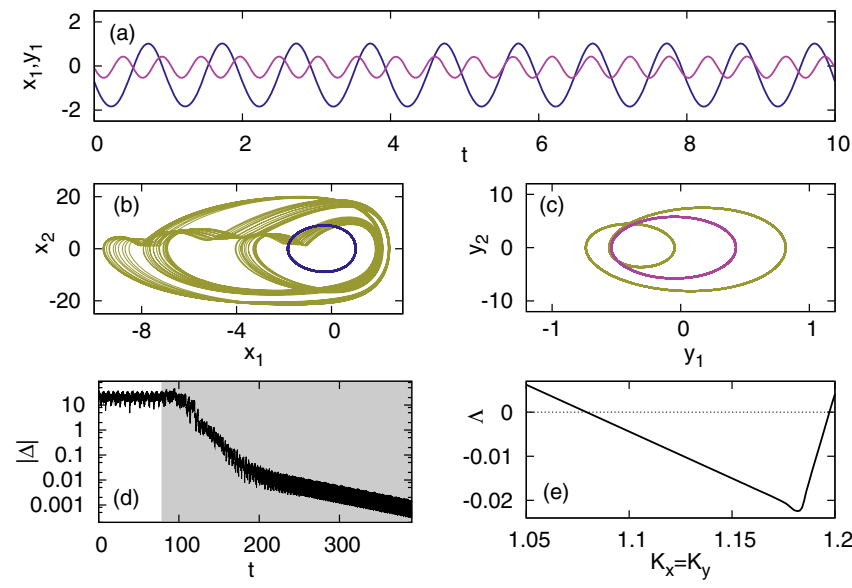

FIG. 2 (color online). Numerical simulation of the Toda system, Eqs. (5). (a) Time traces of the synchronized state for $K_{x}=$ $K_{y}=1.15$ (for other parameter values, see the text). (b),(c) The motion in the phase plane of each oscillator without control (bronze) and with control (blue and red). (d) Time dependence of the residual signal. The shaded region indicates the period where control $K_{x}=K_{y}=1.15$ is applied. (e) Dependence of the leading nontrivial Lyapunov exponent on the control gain as estimated from the exponential decay of the residual control signal (the two vanishing Lyapunov exponents are not displayed). success does not depend on a particular resonance condition between the periods of the orbits and thus stabilizes a proper torus solution.

To test the robustness of the scheme and its implementability in real-world systems, we performed experiments in nonlinear electronic oscillators. The experimental system comprises two diode oscillators coupled via digital delay lines. Details of the electronic oscillators and the delay lines can be found in [13]. The dynamics of each uncoupled oscillator is similar to the dynamics of the Rössler system. Each oscillator has three effective degrees of freedom which are time-dependent voltages, $\left(x_{1}, x_{2}, x_{3}\right)$ and $\left(y_{1}, y_{2}, y_{3}\right)$. The oscillators are designed to be identical within the tolerances of the individual elements in the order of $1 \%$. A sufficient degree of diversity can be achieved by detuning a single parameter. By increasing the parameter of the individual uncoupled oscillators, their dynamics undergoes a bifurcation route starting from a trivial steady state, through a Hopf bifurcation into a periodic orbit and through a Feigenbaum cascade of period-doubling bifurcations into chaos.

As for the coupling we choose $x_{2}(t)$ and $y_{2}(t)$ to be transmitted via the delay lines, so that the control signals $\Delta_{x}(t)=x_{2}(t)-x_{2}(t-\tau)$ and $\Delta_{y}(t)=y_{2}(t)-y_{2}(t-\theta)$ are injected in the input of the corresponding component in the opposite oscillator, respectively. Thus, we have realized a coupling according to Eq. (1) with two $3 \times 3$ coupling matrices containing a single nonvanishing element on the diagonal which, for simplicity, we call $K_{x}$ and $K_{y}$ again. The setup allows for an automatic setting of $K_{x}, K_{y} \in[-1,1]$ in units of the time constant $\omega_{0}=10 \mathrm{kHz}$ of the circuits.

With different combinations of the parameters, we search for mutual stabilization of unstable orbits. As a first observation, we find that for identical systems stabilization is not achieved, in accordance with our theoretical arguments. As revealed by our previous analytic considerations, cf., e.g., Fig. 1, constraints on the size of the Lyapunov exponent are expected for successful control. Hence, we set the control parameter of one oscillator slightly above the point of the supercritical Hopf bifurcation, so that the dynamics without control shows a periodic orbit and the fixed point at the origin is unstable. The parameter of the other system is set above the first period-doubling bifurcation, with dynamics showing a stable period-two orbit spreading around the unstable period one. In the absence of control, the exponential growth rate of transients starting close to these unstable objects reveals their instability, which for the steady state is about 2 orders of magnitude lower than for the period-one orbit. The target of our control is the joint orbit consisting of the unstable fixed point and the unstable period-one orbit, see Fig. 3 (top row). The delay corresponding to the signal from the periodic orbit is set to its period, $\theta=T_{1}$, while for the 
other delay time we choose $\tau \approx T_{1} / 6$. In the $K_{x}-K_{y}$ control parameter plane, the control domain is located in the region of $K_{x} K_{y}<0$, similar to the findings by the simple analytic argument above. We restrict our experiment to only one of the two possible combinations of the coupling gains, because the dynamics showed to be experimentally indistinguishable when the signs of the gains are switched. This is consistent with our theoretical considerations where we found that stability does not depend on the individual control gains but on the product $K_{x} K_{y}$.

For fixed parameters and delay times, we scan the parameter plane of the coupling gains. To set well-defined initial conditions, we implemented for each pair $\left(K_{x}, K_{y}\right)$ a sequence of gain parameters that first lead to successful control of the unstable orbits, so that the trajectories start from well defined initial conditions close to the target state. After transients decay, we record simultaneously the signals $x_{2}(t)$ and $y_{2}(t)$, as well as the outputs of the delay lines $\Delta_{x}(t)$ and $\Delta_{y}(t)$. The measured time series indicates the smallness of the control signals when successful control is applied, see Fig. 3 (middle row).

Figure 3 (bottom row) shows the root mean square of the control signals over the parameter plane. Overall, the hyperbolic shape of the dominant bifurcation structures proves the relevance of the product $K_{x} K_{y}$ for the control
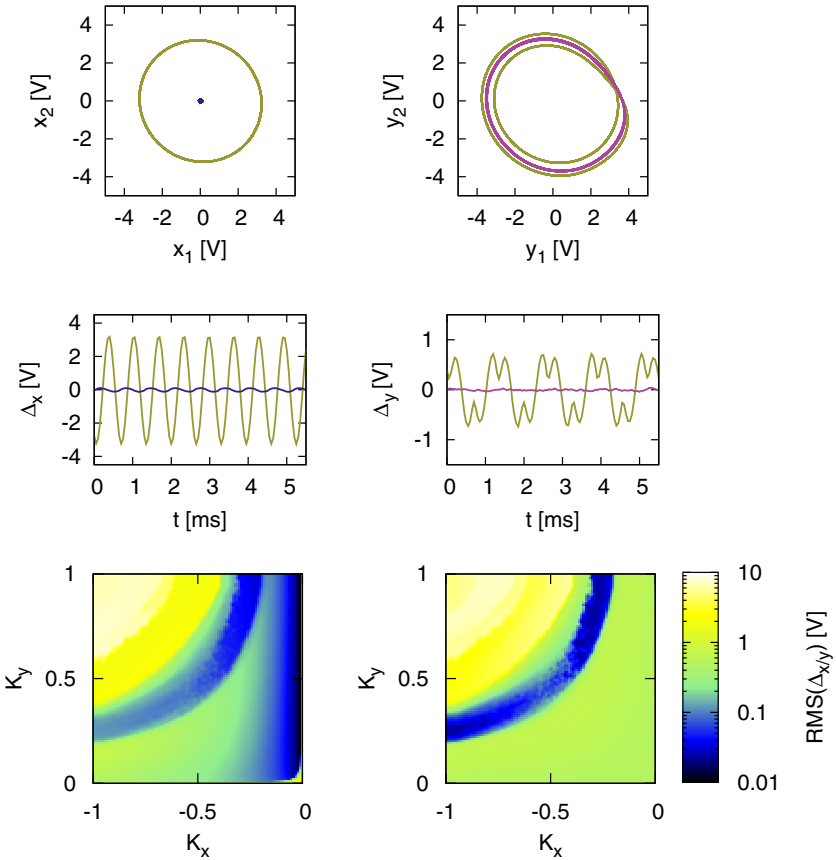

FIG. 3 (color online). Experimental data from coupled electronic circuits: left, oscillator close to the Hopf bifurcation; right, oscillator close to the flip bifurcation. Top, projection of the motion to the phase plane without control (bronze) and with control $K_{x}=-0.27, K_{y}=0.92$ (blue and red). Middle, time traces of the control signal without (bronze) and with control (blue and red). Bottom, root mean square of the control signal in dependence of the coupling gains. configuration. The recorded values of the root mean square range over 3 orders of magnitude, where the maximum values of about $10 \mathrm{~V}$ correspond to the size of an uncontrolled chaotic attractor, and the minimum of $10 \mathrm{mV}$ is the noise floor of the setup corresponding to perfect control or an unperturbed stable steady state. Values below $100 \mathrm{mV}$ can be regarded as successful noninvasive control. In particular, with regard to the target of our control, the hyperbolic region around $K_{x} K_{y} \approx-0.25$, in which both difference signals are clearly low, can be accounted for as a region of successful noninvasive control. Interestingly, the quality of control changes along curves of $K_{x} K_{y}=$ const, a feature which is predominately related to the structure of the eigenmodes which are excited by the noise in the system and which are, unlike the Lyapunov exponent $\Lambda$, not an invariant along such a curve.

It is worth mentioning that the experimental results indicate another mutual control phenomenon, which relies on symmetry breaking and goes beyond the presented analytical considerations. For small values of $K_{x}$, apparently, the steady state is controlled with high accuracy, while in the other system the period-one orbit is not controlled. This means that the steady state system sends its control signal $\Delta_{x}(t)$ to the other system with a high gain $K_{y}$, transmits this signal in the neighborhood of the stable period two, and receives an invasive signal scaled down by $K_{x}$. Overall, the feedback still contains enough information to control the steady state. Although the resulting asymmetric control of one unstable object through an uncontrolled counterpart is, in principle, invasive, the resulting control is experimentally of high quality and in terms of remaining difference signals indistinguishable from a noninvasive control.

In conclusion, we have designed and implemented a mutual time-delayed coupling scheme to synchronize heterogeneous systems, e.g., to stabilize a torus solution. Quite counterintuitively, diversity of the constituents is a key for success, and our basic study is supported by analytical arguments, numerical simulations, and a successful experimental case study. The coupled system shows rich dynamical behavior, for instance, rather subtle linear stability properties of quasiperiodic motion and various experimental features beyond the scope of this study. It is rather straightforward to generalize the current setup for complex coupled heterogeneous networks. Thus, the scheme opens up the possibility for a systematic analytical and experimental study of time delay dynamics and synchronization in coupled systems, with related substantial benefit and insight for interacting complex time delay dynamics.

This work was supported by DFG in the framework of SFB 910. T. J. and I. F. acknowledge support by FEDER and MINECO (Spain) via Project No. TEC2012-36335 (TRIPHOP). 
*w.just@qmul.ac.uk

[1] A. Pikovsky, M. Rosenblum, and J. Kurths, Synchronization: A Universal Concept in Nonlinear Sciences (Cambridge University Press, Cambridge, 2003).

[2] M. G. Rosenblum, A. S. Pikovsky, and J. Kurths, Phys. Rev. Lett. 76, 1804 (1996).

[3] M. G. Rosenblum, A. S. Pikovsky, and J. Kurths, Phys. Rev. Lett. 78, 4193 (1997).

[4] K. Josic, Phys. Rev. Lett. 80, 3053 (1998).

[5] K. Pyragas, Phys. Lett. A 170, 421 (1992).

[6] L. M. Pecora, F. Sorrentino, A. M. Hagerstrom, T. E. Murphy, and R. Roy, Nat. Commun. 5, 4079 (2014).

[7] P. Duarte and S. Klein, Commun. Math. Phys. 332, 1113 (2014).
[8] J. Scheuer and A. Yariv, Phys. Rev. Lett. 97, 140502 (2006).

[9] R. Vicente, C. R. Mirasso, and I. Fischer, Opt. Lett. 32, 403 (2007).

[10] I. Kanter, E. Kopelowitz, and W. Kinzel, Phys. Rev. Lett. 101, 084102 (2008).

[11] B. Fiedler, V. Flunkert, M. Georgi, P. Hövel, and E. Schöll, Phys. Rev. Lett. 98, 114101 (2007).

[12] W. Just, T. Bernard, M. Ostheimer, E. Reibold, and H. Benner, Phys. Rev. Lett. 78, 203 (1997).

[13] T. Jüngling, H. Benner, H. Shirahama, and K. Fukushima, Phys. Rev. E 84, 056208 (2011). 